\title{
Interactive Visualization of Weighted Three-dimensional Alpha Hulls
}

\author{
Amitabh Varshney Frederick P. Brooks, Jr. William V. Wright \\ Department of Computer Science \\ University of North Carolina at Chapel Hill \\ Chapel Hill, NC 27599-3175
}

\begin{abstract}
An interactive visualization of weighted three-dimensional $\alpha$-hulls is presented for static and dynamic spheres. The $\alpha$-hull is analytically computed and represented by a triangulated mesh. The entire surface is computed and displayed in real-time at interactive rates. The weighted threedimensional $\alpha$-hulls are equivalent to smooth molecular surfaces of biochemistry. Biochemistry applications of interactive computation and display of $\alpha$-hulls or smooth molecular surfaces are outlined.
\end{abstract}

\section{Introduction}

The $\alpha$-hull has been defined as a generalization of the convex hull of point-sets by Edelsbrunner, Kirkpatrick, and Seidel $[4,3]$. Given a set of points $P$, a ball $b$ of radius $\alpha$ is defined as an empty $\alpha$-ball if $b \cap P=\phi$. For $0 \leq \alpha \leq \infty$, the $\alpha$-hull of $P$ is defined as the complement of the union of all empty $\alpha$-balls [3]. It has been shown that it is possible to compute the $\alpha$-hull of a set of points $P$ from the Voronoi diagram of $P$. For $\alpha=\infty$ the $\alpha$-hull over the set of points $P$ is the same as their convex hull. Edelsbrunner and Mücke [5] have defined the $\alpha$-shape over $P$ to be the polytope that approximates the $\alpha$-hull over $P$ by replacing circular arcs of the $\alpha$-hull by straight edges and spherical caps by triangles. An $\alpha$-shape of a set of points $P$ is a subset of the Delaunay triangulation of $P$. Edelsbrunner [3], has extended the concept of $\alpha$-shapes to deal with weighted points (i.e. spheres with possibly unequal radii) in three dimensions. An $\alpha$-shape of a set of weighted points $P_{w}$ is a subset of the regular triangulation of $P_{w}$.

The smooth molecular surface of a molecule is defined as the surface which an exterior probe-sphere touches as it is rolled over the spherical atoms of that molecule. This definition of a molecular surface was first proposed by Richards [8]. This surface is useful in studying the structure and interactions of proteins, in particular for attacking the proteinsubstrate docking problem. The analytic computation of the molecular surface was first done by Connolly [2].

Looking at the above definitions, one can see that the weighted $\alpha$-hulls for three dimensions and the smooth molecular surface of a molecule with a probe-radius $\alpha$ have the same definitions. In this video we present the visualization of weighted $\alpha$-hulls as used to define molecular surfaces.

\section{Our Approach}

Computation of $\alpha$-hulls and $\alpha$-shapes has traditionally been done by first constructing power diagrams or regular triangulations. Since these methods involve computing the entire diagram or triangulation first and then culling away the parts that are not required, their complexity is $O\left(n^{2}\right)$ in time, where $n$ is the number of points. This is worst-case optimal, since an $\alpha$-shape in three dimensions could have a complexity of $\Omega\left(n^{2}\right)$.

However, when $\alpha$-hulls are used as molecular surfaces, one can do better. Let $M=\left\{S_{1}, \ldots, S_{n}\right\}$, be a set of spheres, where each sphere, $S_{i}$, is expressed as a pair $\left(c_{i}, r_{i}\right)$, $c_{i}$ being the center of the sphere and $r_{i}$ being the radius of the sphere. Collections of spheres representing molecules have two interesting properties: (i) the minimum distance $d_{i j}$ between any two centers $c_{i}$ and $c_{j}$ is greater than or equal to a positive constant $l_{\text {min }}$ — the smallest bond-length in the molecule and (ii) the values of all the radii can be bounded from above and below by strictly positive values, $0<r_{\min } \leq r_{i} \leq r_{\max }$. We take advantage of the first property to arrive at better running times for our algorithm. Stated simply, the first property says that the number of neighboring atoms within a fixed distance from any atom $i$, is always bounded from above by a constant $k_{\text {max }}$ that depends on the minimum spacing between any two atoms. We refer to two atoms $i$ and $j$ as neighboring if it is possible to place a probe sphere such that it is contact with both $S_{i}$ and $S_{j}$.

A power cell (as described by Aurenhammer [1]) for a given sphere $S_{i}$ with respect to $M$ can be computed as the intersection of $n-1$ halfspaces, each halfspace contributed by the pair $\left(S_{i}, S_{j}\right), 1 \leq j \leq n, j \neq i$. If the average number of neighbors for an atom is $k$, then for our purposes it is sufficient to just compute an approximation to the power cell, which we call a feasible cell, as an intersection of $k$ halfspaces (one halfspace contributed by each neighbor). This can be done in deterministic time $\mathrm{O}(k \log k)$. For $n$ atoms, this task can be parallelized over $n$ processors, each processor computing the feasible cell for one atom. To check if a feasible cell is non-null, we use a randomized linear programming algorithm that has linear expected time complexity and is quite fast in practice [9]. For details of our approach the interested reader can refer to [10]. 
We have used ideas from the theory of packing and covering of spheres to estimate the value for $k$, the average number of neighboring atoms, for an atom in protein molecules. We can prove that for proteins, $k<140$ for a probe-sphere radius of $1.4 \AA$ - the radius of a water molecule. Details about this result can be found in [11]. In practice we have found that for protein molecules and for a probe-radius of $1.4 \AA, k$ is around 45

In the algorithms for computing the convex hull of a set of points, it is assumed that the points are in a general position, i.e. no more than $d$ points lie on the same $d-1$ dimensional hyperplane. In reality this assumption often fails to hold, leading to problems. For example, planar benzene rings occur often in proteins, causing six carbon and six hydrogen atoms to be all coplanar. One of the recent approaches to solving this problem has been to perturb the input point set slightly to avoid these degeneracies. The approach of Emiris and Canny [6] perturbs the $j^{\text {th }}$ dimension of the $i^{\text {th }}$ point as:

$$
p_{i, j}(\epsilon)=p_{i, j}+\epsilon\left(i^{j} \bmod q\right) 1 \leq i \leq n, 1 \leq j \leq d
$$

where $\epsilon$ is a symbolic infinitesimal and $q$ is the smallest prime greater than $n$. Instead of performing exact integer arithmetic, we just perturb the centers of the spheres by the above scheme and that has worked quite well for us in practice.

With no preprocessing, we can compute the molecular surface for a 396 atom Crambin and a probe-radius of $1.4 \AA$, using 40 Intel $\mathrm{i} 860$ processors in 0.2 seconds. We have implemented $\alpha$-hulls on Pixel-Planes 5 [7], though the method is general enough to be easily implemented on any other parallel architecture. Our approach is analytically exact. The only approximation is the degree of tessellation of spherical and toroidal patches.

\section{Applications in Biochemistry}

Interactive computation and display of molecular surfaces should benefit biochemists in three important ways. First, the ability to change the probe-radius interactively helps one study the surface. Second, it helps in visualizing the changing surface of a molecule as its atom positions are changed. These changes in atom positions could be due to user-defined forces as the user attempts to modify a molecular model on a computer. Third, it assists in incorporating the effects of the solvent into the overall potential energy computations during the interactive modifications of a molecule on a computer.

\section{Scope for further work}

At present we are not using any incremental temporal information in constructing these surfaces. Thus, if the atoms move slightly from their positions, the whole surface is recomputed from the beginning, although one could conceivably store the feasible cell description with each atom. Assuming the atoms of the molecule move along continuous trajectories, it should be possible to compute such surfaces (and indeed $\alpha$-hulls and $\alpha$-shapes) incrementally and efficiently by using the information from previous time steps.

\section{Acknowledgements}

We would like to acknowledge the valuable discussions we had with Pankaj K. Agarwal, Dinesh Manocha, Jan F. Prins, and David C. Richardson during various stages of this work We would also like to acknowledge Michael E. Pique and Victoria Roberts at the Scripps Clinic for the molecular dynamics data. We would like to thank the anonymous referees for their suggestions which have led to improvements in the presentation of this extended abstract as well as the video. This work was supported by NIH National Center for Research Resources grant number 5-P41-RR02170.

\section{References}

[1] F. Aurenhammer. Power diagrams: Properties, algorithms and applications. SIAM Journal of Computing, $16(1): 78-96,1987$

[2] M. L. Connolly. Analytical molecular surface calculation. Journal of Applied Crystallography, 16:548-558, 1983 .

[3] H. Edelsbrunner. Weighted alpha shapes. Technical Report UIUCDCS-R-92-1740, Department of Computer Science, University of Illinois at Urbana-Champaign, 1992.

[4] H. Edelsbrunner, D. G. Kirkpatrick, and R. Seidel. On the shape of a set of points in the plane. IEEE Transactions on Information Theory, IT-29(4):551-559, 1983.

[5] H. Edelsbrunner and E. P. M"ucke. Three-dimensional alpha shapes. ACM Transactions on Graphics, 13(1), 1994.

[6] I. Emiris and J. Canny. An efficient approach to removing geometric degeneracies. In Eighth Annual Symposium on Computational Geometry, pages 74-82, Berlin, Germany, June 1992. ACM Press.

[7] H. Fuchs, J. Poulton, J. Eyles, T. Greer, J. Goldfeather, D. Ellsworth, S. Molnar, G. Turk, B. Tebbs, and L. Israel. Pixel-planes 5: A heterogeneous multiprocessor graphics system using processor-enhanced memories. In Computer Graphics: Proceedings of SIGGRAPH'89, volume 23, No. 3, pages 79-88. ACM SIGGRAPH, 1989.

[8] F. M. Richards. Areas, volumes, packing and protein structure. Ann. Rev. Biophys. Bioengg., 6:151-176, 1977 .

[9] R. Seidel. Linear programming and convex hulls made easy. In Sixth Annual ACM Symposium on Computational Geometry, pages 211-215, Berkeley, California, June 1990. ACM Press.

[10] A. Varshney and F. P. Brooks, Jr. Fast analytical computation of Richards's smooth molecular surface. In G. M. Nielson and D. Bergeron, editors, IEEE Visualization '93 Proceedings, pages 300-307, October 1993.

[11] A. Varshney, W. V. Wright, and F. P. Brooks, Jr. Bounding the number of unit spheres inside a larger sphere. Technical Report UNC-CS-TR-93-039, Department of Computer Science, University of North Carolina at Chapel Hill, 1993. 